Revue internationale de l'économie sociale

Recma

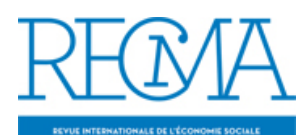

\title{
La législation sur les coopératives de crédit en Espagne
}

\section{Gemma Fajardo García}

Numéro 323, janvier 2012

URI : https://id.erudit.org/iderudit/1018340ar

DOI : https://doi.org/10.7202/1018340ar

Aller au sommaire du numéro

Éditeur(s)

Association Recma

ISSN

1626-1682 (imprimé)

2261-2599 (numérique)

Découvrir la revue

Citer cet article

Fajardo García, G. (2012). La législation sur les coopératives de crédit en

Espagne. Revue internationale de l'économie sociale, (323), 24-28.

https://doi.org/10.7202/1018340ar d'utilisation que vous pouvez consulter en ligne.

https://apropos.erudit.org/fr/usagers/politique-dutilisation/ 


\title{
LA LÉGISLATION SUR LES COOPÉRATIVES DE CRÉDIT EN ESPAGNE
}

\author{
par Gemma Fajardo García*
}

* Professeur de droit commercial, université de Valence (Espagne). Mél.: Isabel.Fajardo@uv.es.

(1) Ce travail est le résultat d'un projet de recherche financé par le ministère espagnol de l'Education et de la Science, "Nueva etapa en la modernización del derecho de sociedades europeo" (SEJ 2007$62969 / J U R I)$. II a donné lieu à une communication lors du colloque européen du comité de recherche de I'ACl organisé à l'université Lumière-Lyon 2 du 2 au 4 septembre 2010, dont est tirée cette étude.
$E$ n 2010, le système bancaire espagnol comptait 160 banques résidentes, 36 caisses d'épargne et 80 coopératives de crédit. Les coopératives de crédit sont définies par la législation comme des sociétés dont l'objet social est de répondre aux besoins financiers de leurs associés et des tiers par le biais de l'exercice des activités spécifiques des établissements de crédit. En Espagne, elles se répartissent entre les caisses rurales et les caisses populaires et professionnelles, selon la composition de leur sociétariat et leur but poursuivi. Au fil de leur histoire, le jeu des fusions a engendré une réduction du nombre des entités, qui est passé de plus de 1000 au début de la Guerre civile (1936) à 80 en 2010. Cette réduction est due aux transformations des coopératives de crédit en sections de crédit, aux absorptions par des coopératives, des banques et des caisses d'épargne, aux fusions, aux transformations et aux liquidations ${ }^{(1)}$.

\section{Bref historique du crédit coopératif en Espagne}

(2) Les caisses rurales sont regroupées dans la Cajas Rurales Asociadas (CRA), qui n'est pas vraiment un groupe d'entreprises, mais seulement un ensemble de partenariats économiques.
Les premières coopératives de crédit apparaissent en Espagne au cours de la seconde moitié du XIX ${ }^{\mathrm{e}}$ siècle (Cooperativa Papeleros de Buñol, à Valence, en 1858), et les premières caisses rurales au début du $\mathrm{Xx}^{\mathrm{e}}$ siècle, avec la Caja Rural San Isidro de Castellón (1903), qui est toujours en activité. Les caisses populaires et professionnelles se développent dans les années 60 (Caja Laboral Popular de Mondragón, Caja Ingenieros de Barcelona, Caja Caminos ou Caja Grume co). A la même époque, les coopératives de crédit sont intégrées au système bancaire (Ley de Bases de Ordenación del Crédito y la Banca de 1962) et sont assujetties au Banco de España (1971). L'Unión nacional de cooperativas de crédito (Unacc) est créée en 1969. Avec la crise des caisses rurales (1983-1987) commence à proprement parler le regroupement des coopératives de crédit au sein de l'Unacc ${ }^{(2)}$. Tout d'abord, un accord d'association est signé entre le Banco de Crédito Oficial et 57 caisses rurales, donnant naissance au Grupo Asociado BCECajas Rurales. A partir de 1987, différentes caisses rurales quittent le groupe pour constituer en 1989, avec le Deutsche Genossenschaftsbank (DGBank), le Banco Cooperativo Español.

En 1989, l'Asociación Española de Cooperativas de Crédito (Asociación Española de Cajas Rurales à compter de 1995) est créée, englobant la 
quasi-totalité des caisses rurales. Cette association deviendra le cœur du futur Grupo Caja Rural (GCR), auquel seront intégrés le Banco Cooperativo Español et d'autres entités créées dans les années 80 par le Grupo Asociado BCE-Cajas Rurales, comme le Rural Servicios Informáticos (RSI) et le Rural Grupo Asegurador (RGA). Aujourd'hui, 72 des 76 caisses rurales font partie du Groupe Caisses rurales (GCR).

\section{Structure du crédit coopératif en Espagne en 2010}

La plupart des coopératives de crédit sont des caisses rurales (76 sur 80 ). Des coopératives de crédit ont été plus tardivement constituées par des collectifs de professionnels (ingénieurs, avocats, professeurs, etc.). D’autres apparaissent comme des éléments intégrés à un groupe coopératif d'entreprises. C'est le cas de la Caja Laboral Popular (Grupo Mondragón) ou de la Caixa Popular (Grup Cooperatiu Valenciá).

Toutes les coopératives de crédit se rassemblent au sein de l'Unión Nacional de Cooperativas de Crédito et la quasi-totalité des caisses rurales appartiennent à l'Asociación Española de Cajas Rurales, exception faite de la Caja Mar et d'Ipar Kutxa. De plus, les coopératives de crédit sont en marge des structures fédératives des coopératives ou de l'économie sociale (la Cepes, par exemple).

\section{Les modalités de création et de fonctionnement des coopératives de crédit}

En tant qu'établissements de crédit, les coopératives sont régies par la réglementation bancaire (loi 26/1988; loi 13/1985; RD 2606/1996). La constitution d'une coopérative de crédit requiert une autorisation préalable du ministère de l'Economie et des Finances, après émission d'un rapport de la Banque d'Espagne. La demande de constitution doit être

\section{Tableau 1}

La coopération bancaire espagnole en chiffres (2010)

\begin{tabular}{|l|r|r|r|r|r|}
\hline & Entreprises & \multicolumn{1}{|l|}{ Employés } & \multicolumn{1}{l|}{ Agences } & \multicolumn{1}{l|}{ Crédits } & \multicolumn{1}{l|}{ Dépôts } \\
\hline $\begin{array}{l}\text { Coopératives } \\
\text { de crédit }\end{array}$ & 80 & 20545 & 5018 & 96802 & 91161 \\
\hline $\begin{array}{l}\text { Caisses } \\
\text { d'épargne }\end{array}$ & 36 & 125162 & 22649 & 848348 & 719320 \\
\hline Banques & 160 & 111871 & 15227 & 837140 & 623541 \\
\hline
\end{tabular}


signée par un groupe de promoteurs composé, au moins, de cinq personnes morales exerçant l'activité propre à leur objet social de façon ininterrompue, pendant au moins deux années avant la constitution, ou de cent cinquante personnes physiques. S'il s'agit d'une caisse rurale, le groupe promoteur doit comprendre au moins une coopérative agricole ou cinquante personnes physiques associées propriétaires d'exploitations agricoles. Le capital social minimal nécessaire pour constituer une coopérative de crédit est déterminé par le gouvernement, après élaboration d'un rapport de la Banque d'Espagne et en fonction du cadre territorial et du nombre total d'habitants correspondant. Tous les associés doivent posséder au moins un titre nominatif d'apport. Les statuts établissent la valeur de l'apport - cette dernière ne pouvant pas être inférieure à 60,10 euroset le nombre minimal de titres que doivent posséder les associés. Tous les titres ont la même valeur nominale. La condition d'associé n'est pas perdue lorsque la valeur nominale des apports est annulée ou réduite en cas d'assainissement, approuvé par le fonds de garantie de dépôts, ou de pertes. Le montant total des apports de chaque associé ne peut pas excéder $20 \%$ du capital social s'il s'agit d'une personne morale et 2,5\% s'il s'agit d'une personne physique. Les personnes morales ne revêtant pas le caractère de coopératives ne peuvent en aucun cas détenir plus de $50 \%$ du capital de la coopérative.

Leur réglementation sur le plan sociétaire est régie par la législation sur les coopératives, dont la compétence est partagée, en Espagne, entre l'Etat et les communautés autonomes. L'Etat détient la compétence exclusive pour réglementer les bases de crédit et la banque, tandis que les communautés autonomes ont la compétence exclusive de la réglementation des coopératives. La loi 13/1989 sur les coopératives de crédit et son règlement (RD 84/1993) ont qualité de réglementation de base dans leur intégralité et relèvent de ce fait de la compétence de l'Etat. Cette qualification a été ratifiée par le Tribunal Constitucional et a retiré aux communautés autonomes, de facto, toute compétence en matière de développement. En fonction de l'étendue de l'activité de la coopérative à l'intérieur ou en dehors d'une communauté autonome, la législation de l'Etat ou celle de la communauté autonome sera également applicable. Les coopératives de crédit peuvent réaliser les mêmes opérations actives, passives et de services que les autres établissements de crédit, mais en accordant une attention préférentielle aux besoins de leurs associés. L'ensemble des opérations actives avec des tiers ne peut dépasser $50 \%$ de l'ensemble des fonds de l'entité.

\section{L'impact de la législation sur le développement du crédit coopératif}

La législation favorise l'existence de coopératives de crédit à la fois fortes et compétitives, offrant des garanties et de la rentabilité aux tiers et à leurs associés. Elle propose des avantages fiscaux en échange de la limitation et de la mise en place des conditions quant à leurs opérations, mais cela 
(3) Soler F., 2002, "La identidad cooperativa como garantía de futuro: las cooperativas de crédito ante la trivialización de sus principios" Ciriec-España, Revista de Economía Política, Social y Cooperativa, $\mathrm{n}^{\circ} 40$.

(4) Minguez F., 2010, « Estructuras de integración contractuales entre entidades de crédito: los denominados Sistemas Institucionales de Protección ", Revista de Derecho del Mercado de Valores, $n^{\circ} 7$. ne semble constituer ni un grand avantage ni un grand préjudice pour les coopératives de crédit. La législation permet tout d'abord des opérations actives avec des tiers jusqu'à $50 \%$, mais il ne faut pas séparer les résultats. Elle permet aussi le vote plural, proportionnel aux apports au capital social. La coopérative doit allouer au fonds de reserve obligatoire au moins $20 \%$ des excédents disponibles, qui ne pourront pas être distribués, et au moins $10 \%$ au fonds d'éducation et de promotion coopérative.

\section{Remise en cause de la gestion démocratique}

Si la législation maintient à l'origine la présence des principes coopératifs, elle tend à limiter de plus en plus leur portée. Les cas les plus frappants enfreignent le principe de gestion démocratique en raison de l'admission d'un vote plural possiblement proportionnel au capital social ${ }^{(3)}$. De plus, s'impose désormais un système d'élection d'administrateurs au moyen de listes fermées qui ne peuvent être proposées que par le conseil de direction ou un nombre élevé d'associés, difficile à atteindre.

Enfin, l'attention doit être portée sur la plus récente des réglementations en matières de groupe, de fusion et de transformation. L'Espagne est en effet immergée dans un processus de restructuration de son secteur bancaire. Elle mène actuellement, à la demande de la Banque d'Espagne, des processus d'intégration dénommés systèmes institutionnels de protection (SIP), qui affectent les caisses d'épargne et les coopératives de crédit ${ }^{(4)}$. Le SIP doit permettre l'intégration de l'activité économique tout en préservant la personnalité juridique et la raison sociale.

\section{Le risque de banalisation}

Ces processus risquent de transformer les coopératives de crédit, mais aussi les caisses d'épargne, en banques. L'entité centrale du SIP doit en effet être l'un des établissements de crédit composant le système ou un établissement de crédit dans lequel chacun d'entre eux détient une participation. La durée minimale du SIP est de dix ans; tous les établissements associés mettent en commun au moins $40 \%$ de leurs fonds propres et l'entité centrale déterminera les politiques et les stratégies de l'activité, ainsi que les niveaux et les mesures de contrôle interne et de gestion des risques. Les caisses d'épargne doivent choisir l'une d'entre elles en tant qu'entité centrale ou faire le choix de constituer une banque exerçant ces fonctions. A la fin de 2011, sur les 35 caisses d'épargne, 24 opèrent par le biais d'une banque.

Les coopératives de crédit sont donc tentées de créer une banque pour être à la tête du groupe du SIP. Avec le temps, une fusion de toutes ces coopératives de crédit au sein de la banque ainsi créée est probable. Cela entraînerait, de fait, la disparition des coopératives en tant que telles, même si elles maintiennent leur dénomination en tant que marque, comme le font les caisses d'épargne.

Par ailleurs, la réforme de la législation espagnole au sujet des modifications structurelles des sociétés (loi 3/2009) a introduit dans la loi sur les 
(5) Fajardo I. G., 2010, « Aspectos de la transformación de las cooperativas de crédito tras la Ley de modificaciones estructurales de 2009 ", Estudios de Derecho del Mercado Financiero, homenaje al Profesor Vicente Cuñat Edo, Universitat de València. coopératives de crédit la possibilité de leur transformation en une autre forme sociale. En cas de transformation, leur fonds de réserve obligatoire sera intégré au capital social de l'entité résultant de la transformation. Si cette norme est interprétée conformément au débat parlementaire qui lui a donné naissance, les sociétaires, titulaires de parts d'un minimum de 60,10 euros, verraient augmenter la valeur de celles-ci à hauteur du montant des réserves, qui jusqu'alors ne pouvaient pas être réparties en cas de transformation de la coopérative en banque. La mesure légale semble appelée à provoquer la transformation de coopératives de crédit en banques ${ }^{(5)}$.

\section{Conclusion}

Si la législation réaffirme les grands principes coopératifs, elle y ajoute de plus en plus de limites. Le principe de la porte ouverte a été remis en cause par le décret royal 1309/2005, selon lequel le remboursement des apports au capital des associés qui se sont séparés pourra être inconditionnellement refusé par le conseil de direction. Lavenir des coopératives de crédit semble ainsi d'autant plus incertain que la loi 13/2009 encourage leur transformation en banques classiques. 